\title{
Attended and unattended products direct buying options using the same neural circuits
}

\author{
Alvaro Machado Dias* \\ Department of Psychiatry, Neuroimage Lab, University of São Paulo, São Paulo, Brazil \\ *Correspondence: alvaromd@usp.br
}

In this article, I will review and discuss some interesting findings regarding neural predictors of decision-making.

Most models of preference which are applied to consumer behavior rely on the assumption that information processing occurs on demand and that the mental dynamics which culminate in a purchase involve weighing up options and subsequently engaging in reasoning processes that will lead to decision-making (for further discussion, see Dabholkar, 1994). Conversely, the ability to predict consumer behavior based on the selective activation of certain brain circuits prior to the conscious evaluation of the product and the onset of deliberative intentions represents a breakthrough for the new science of neuromarketing and for the philosophy of mind. This perspective challenges the concept of free will by suggesting that it is possible for an observer to know more about a subject's future actions than the subject him or herself.

The process of evolution that has led to this idea began with earlier studies on neural predictors of decision-making (e.g., Knutson et al., 2001). These predictors were first applied to consumer behavior when Knutson et al. (2007) ran logistic regressions that revealed that the activation of certain brain circuits (which had been linked to preference toward a certain product (nucleus accumbens) and calculations regarding the fairness of its price [insula and the mesial prefrontal cortex]) could be used in order to predict purchase decisions in the SHOP experimental paradigm, which involves consecutive buying decisions. More recently, Tusche et al. (2010) created an experimental paradigm involving consecutive choice that allowed for the comparison of neural predictors of buying behavior for goods to which participants had paid low and high levels of attention.

In the condition where the participants had paid the most attention, 17 males interested in automobiles were presented with pictures of real cars and were required to judge their attractiveness, while functional neuroimaging was conducted using a 3-Tesla scanner. In the low attention condition, 15 males with the same profile were required to engage in a distracting task, the aim of which was to divert their attention away from the images of cars that were simultaneously presented in their peripheral field of vision. In both conditions, brain scanning was conducted while the participants remained unaware of the fact that they were going to be asked to make a consumer decision.

The decision-making task followed the brain scans and involved two procedures: all participants were asked to define their willingness to buy each of the vehicles (their options were: No/Yes/Not sure), while participants in the second condition also had to rate the attractiveness of each car. By this means, parameters of choice/no choice were defined for both groups, making it possible to establish the extent to which willingness to buy could be reduced to a judgment of attractiveness.

The authors adopted a multivariate data classification procedure (support vector machine) to their imaging analysis. This method involves full spatial patterning of brain activity, measured simultaneously at many locations (Haynes and Rees, 2006). This method means that it is possible to account for spatial correlations among voxels that are not part of a limited neighborhood, as a result of replacing predefined reference functions with a model-free algorithm (for further discussion, see Wang et al., 2007).

Multivariate data classification has several benefits compared to the conventional univariate approach. For instance, while the BOLD signal related to single site activation is often too weak to allow the establishment of a statistically valid association between increased activity in a particular location in the brain and a certain task or mental state, multivariate analysis allows greater sensitivity, by taking the view that signal may accumulate along several sites (Haynes and Rees, 2006). In that same vein, this method enables a greater understanding of complex brain processes that rely on the simultaneous activation of more than one brain site and, in the opposite direction, the discrimination of core brain networks related to different experimental conditions, in cases where overlapping activity in specific locations could potentially adversely affect conclusions about their overall equivalence (Peelen and Downing, 2007).

One of the main conclusions of this experiment was that the activation of certain brain areas could be used to predict buying decisions in both experimental conditions. This was most straightforward in terms of the activation of the insula, which showed $72 \%$ decoding accuracy for the high attention group and $73 \%$ for the low attention group. In addition, the activation of the medial prefrontal cortex (MPFC), which revealed $73 \%$ and $76 \%$ decoding accuracy for the high/low attention groups respectively, also had common predictive capabilities.

However, it is important to note that the division of the MPFC into smaller areas (as was performed in this study) showed that the key areas engaged in cognitive processes that led to predictability for the high attention group were found in the ventral MPFC, while areas in the left MPFC were found to have a similar role in the low attention group. Moreover, it is worth noting that the right dorsal MPFC showed 75\% decoding accuracy for the high attention group and no decoding accuracy for the low attention group, leading to the conclusion that the overlap between predictive brain activation in both conditions was partial rather than complete in nature.

Another key conclusion of this study is that buying decisions are processed independently from attractiveness, as evaluated using the high attention group. Attractiveness was harder to predict, but 
showed greater predictability in relation to activation patterns in the right middle frontal gyrus (47\% decoding accuracy).

The conclusions drawn from the neuroimaging contradict previous studies on neural predictors of purchase (Knutson et al., 2007), monetary rewards (Knutson et al., 2001), and financial decisions (for a review, see Knutson and Bossaerts, 2007) to a certain extent, since these studies state that the striatal dopaminergic circuits have a role in these kinds of decision-making processes. Such a conclusion was absent from Tusche's et al. (2010) findings.

The divergence in neuroimaging results between this and previous studies seems to have emerged from two methodological differences. First, Knutson's aforementioned experiments used a standard activation-based analysis approach whereas Tusche et al. used a pattern classification approach. Second, Tuche et al.'s study did not include prices associated with the cars' images, as Knutson et al.'s (2007) did, which is expected to have induced participants to engage in a two-stage mental operation, first by attending to the goods and then by evaluating the fairness of the prices. In other words, information processing in the context of Tusche et al.'s study includes both positive and negative aspects of the goods, contrary to what seems to occur in Knutson et al.'s. This is in line with the findings from Knutson et al. (2007), who stated that "NAcc activation correlated strongly with product preference" (p. 153), as well as with Tusche et al.'s conclusions on the independence of brain activation related to general attractiveness and willingness to buy.

It is worth noting that this difference does not necessarily represent a limitation of the current study. Insofar as experiments regarding consumer behavior try to advance the understanding of real-life situations, this study provides further knowledge regarding marketing strategies, which usually involve attempts to expose potential consumers to images of goods without price tags, so as to improve the product's attractiveness. By taking these methodological differences into account, we find that the few available studies agree on the inclusion of the activation of the MPFC and the insula among the neural predictors of buying (Knutson and Bossaerts, 2007; Knutson et al., 2007; Tusche et al., 2010).

This study also sheds light on a very interesting philosophical aspect, related to the fact that the process of paying attention to images of goods carries enough clues to predict further economic decisions (namely willingness to buy). It may therefore be assumed that these images were inherently processed as elements of an economic logic, operating independently from simple attraction.

Such a perspective opens several lines of exploration for further research, based on similar experimental paradigms. For instance, it would be very interesting to know whether neural predictability is constant when there is a strong preference bias e.g., through recreating Tusche et al.'s study with images of automobiles that tend to fascinate car lovers, like Ferraris, Lamborghinis, and Rolls Royces (one could hypothesize that these images would lead to much lower levels of insula activation). It would also be interesting to know if it is still possible to establish neural predictors of buying decisions when dealing with goods whose prices are less clearly defined (e.g., unknown art pieces). This last proposal may be of particular interest in light of the wellknown anchoring and adjustment effects that tend to shape the establishment of utility values for goods whose prices are initially unknown by the experimental population (Northcraft and Neale, 1987).

Finally, this study suggests that market researchers should reconsider the actual cost/ benefit of publicity campaigns that bombard potential consumers with a product's image, in opposition to more disseminated and near-subliminal promotional strategies. This is clearly not at the heart of the experiment being discussed, but it is one of its most direct and immediate applications.

\section{ACKNOWLEDGMENT}

This work was supported by Fundação de Amparo à Pesquisa do Estado de São Paulo, FAPESP, Brazil.

\section{REFERENCES}

Dabholkar, P. A. (1994). Incorporating choice into an attitudinal framework: analyzing models of mental comparison processes. J. Consum. Res. 21, 100-118.

Haynes, J.-D., and Rees, G. (2006). Decoding mental states from brain activity in humans. Nat. Rev. Neurosci. 7, 523-534.

Knutson, B., Adams, C. M., Fong, G. W., and Hommer, D. (2001). Anticipation of increasing monetary reward selectively recruits nucleus accumbens. J. Neurosci. $21,159$.

Knutson, B., and Bossaerts, P. (2007). Neural antecedents of financial decisions. J. Neurosci. 27, 8174-8177.

Knutson, B., Rick, S., Wimmer, G. E., Prelec, D., and Loewenstein, G. (2007). Neural predictors of purchases. Neuron 53, 147-156.

Northcraft, G. B., and Neale, M. A. (1987). Experts, amateurs, and real estate: an anchoring-and-adjustment perspective on property pricing decisions. Organ. Behav. Hum. Decis. Process 39, 84-97.

Peelen, M. V., and Downing, P. E. (2007). Using multivoxel pattern analysis of fMRI data to interpret overlapping functional activations. Trends Cogn. Sci. 11, 4-5.

Tusche, A., Bode, S., and Haynes, J.-D. (2010). Neural responses to unattended products predict later consumer choices. J. Neurosci. 30, 8024-8031.

Wang, Z., Childress, A. R., Wang, J., and Detre, J.A. (2007). Support vector machine learning-based fMRI data group analysis. NeuroImage 36, 1139-1151.

Received: 11 September 2010; accepted: 15 October 2010; published online: 11 November 2010.

Citation: Dias AM (2010) Attended and unattended products direct buying options using the same neural circuits. Front. Neurosci. 4:185. doi: 10.3389/fnins.2010.00185 This article was submitted to Frontiers in Decision Neuroscience, a specialty of Frontiers in Neuroscience. Copyright $\odot 2010$ Dias. This is an open-access article subject to an exclusive license agreement between the authors and the Frontiers Research Foundation, which permits unrestricted use, distribution, and reproduction in any medium, provided the original authors and source are credited. 